\title{
A RANDOM FREDHOLM INTEGRAL EQUATION
}

\author{
W. J. PADGETT AND CHRIS P. TSOKOS
}

Abstract. The aim of this paper is the study of a random or stochastic integral equation of the Fredholm type given by $x(t ; \omega)=$ $h(t ; \omega)+\int_{0}^{\infty} k_{0}(t, \tau ; \omega) e(\tau, x(\tau ; \omega)) d \tau, t \geqq 0$, where $\omega \in \Omega$, the supporting set of the probability measure space $(\Omega, A, P)$. The existence and uniqueness of a random solution to the above stochastic integral equation is considered. A random solution, $x(t ; \omega)$, of such a random equation is defined to be a random function which satisfies the equation almost surely. Several theorems and useful special cases are presented which give conditions such that a random solution exists.

1. Introduction. The object of this note is to state some results concerning the existence and uniqueness of a random solution of a stochastic integral equation of the Fredholm type of the form

$$
x(t ; \omega)=h(t ; \omega)+\int_{0}^{\infty} k_{0}(t, \tau ; \omega) e(\tau, x(\tau ; \omega)) d \tau,
$$

where $t \in R_{+}$and

(i) $\omega \in \Omega$, where $\Omega$ is the supporting set of the complete probability measure space $(\Omega, A, P)$;

(ii) $x(t ; \omega)$ is the unknown random function for $t \in R_{+}$, the nonnegative real numbers;

(iii) $h(t ; \omega)$ is the stochastic free term defined for $t \in R_{+}$;

(iv) $k_{0}(t, \tau ; \omega)$ is the stochastic kernel defined for $t$ and $\tau$ in $R_{+}$; and

(v) $e(t, x)$ is a scalar function defined for $t \in R_{+}$and $x \in R$, the real line.

The above equation is a generalization of the equation of Anderson [1] in that the stochastic kernel is a general random function, the equation is nonlinear, and the interval of integration is $R_{+}$. To study existence of a unique random solution of equation (1.1), we first present theorems concerning the existence of a unique random solution of the mixed

Received by the editors June 29, 1971.

AMS 1969 subject classifications. Primary 6075, 6040; Secondary 4511.

Key words and phrases. Stochastic integral equation of Fredholm type, random Fredholm integral equation, random mixed Volterra-Fredholm integral equation, random solutions.

$\stackrel{\bar{c}}{\mathrm{C}}$ American Mathematical Society 1972 
random Volterra-Fredholm equation of the form

$$
\begin{aligned}
x(t ;())= & h(t ; \omega)+\int_{0}^{t} k(t, \tau ; \omega) f(\tau, x(\tau ; \omega)) d \tau \\
& +\int_{0}^{\infty} k_{0}(t, \tau ;(\omega) e(\tau, x(\tau ; \omega)) d \tau, \quad t \geqq 0,
\end{aligned}
$$

where

(vi) $k(t, \tau ; \omega)$ is a stochastic kernel defined for $t$ and $\tau$ satisfying $0 \leqq \tau \leqq t<\infty ;$ and

(vii) $f(t, x)$ is a scalar function of $t \in R_{+}$and $x \in R$.

Then equation (1.1) is obtained from (1.2) when $k(t, \tau ; \omega)$ is equal to zero for all $t$ and $\tau$ such that $0 \leqq \tau \leqq t<\infty$ and almost all $\omega \in \Omega$. Also, the stochastic integral equation of the Volterra type of Tsokos [9] may be obtained from (1.2).

Certain aspects of the "theory of admissibility" as introduced into the theory of deterministic integral equations by Corduneanu [2] and methods of probabilistic functional analysis were used in obtaining the results announced here. Nonstochastic integral equations of the form (1.2) have been studied by Miller, Nohel, and Wong [5], Petrovanu [7], and Cordurieanu [3], among others.

2. Definitions and assumptions. We make the following assumptions concerning the equations (1.1) and (1.2). The random functions $x(t ; \omega)$ and $h(t ; \omega)$ are second order stochastic processes, continuous (in mean square) in $t \geqq 0$; that is, for each $t \in R_{+}, x(t ; \omega)$ and $h(t ; \omega)$ are in the space $L_{2}(\Omega, A, P)$. The functions $f(t, x(t ; \omega))$ and $e(t, x(t ; \omega))$ are functions of $t \in R_{+}$with values in $L_{2}(\Omega, A, P)$. For each $t$ and $\tau$ in $R_{+}$, $k_{0}(t, \tau ; \omega)$ has values in the space $L_{\infty}(\Omega, A, P)$, and the function $k(t, \tau ; \omega)$ for each $t$ and $\tau$ such that $0 \leqq \tau \leqq t<\infty$ has values in $L_{\infty}(\Omega, A, P)$. Thus, $k(t, \tau ; \omega) f(\tau, x(\tau ; \omega)), 0 \leqq \tau \leqq t<\infty$, and $k_{0}(t, \tau ; \omega) e(\tau, x(\tau ; \omega)), t, \tau \in R_{+}$, are in $L_{2}(\Omega, A, P)$.

The mappings

$$
(t, \tau) \rightarrow k(t, \tau ; \omega) \text { and }(t, \tau) \rightarrow k_{0}(t, \tau ; \omega)
$$

from the sets

and

$$
\Delta=\{(t, \tau): 0 \leqq \tau \leqq t<\infty\}
$$

$$
\Delta_{0}=\{(t, \tau): 0 \leqq \tau<\infty, 0 \leqq t<\infty\},
$$

respectively, into $L_{\infty}(\Omega, A, P)$ are continuous. That is,

and

$$
\underset{\omega \in \Omega}{\mathrm{P} \text {-ess } \sup }\left|k\left(t_{n}, \tau_{n} ; \omega\right)-k(t, \tau ; \omega)\right| \rightarrow 0
$$

$$
\underset{\omega \in \Omega}{\text { P-ess sup }}\left|k_{0}\left(t_{n}, \tau_{n} ; \omega\right)-k_{0}(t, \tau ; \omega)\right| \rightarrow 0
$$


as $n \rightarrow \infty$ whenever $\left(t_{n}, \tau_{n}\right) \rightarrow(t, \tau)$ as $n \rightarrow \infty$. Denote the norm of $k_{0}(t, \tau ; \omega)$ in $L_{\infty}(\Omega, A, P)$ for $t, \tau \in R_{+}$by

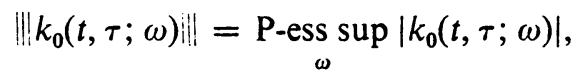

and the norm of $k(t, \tau ; \omega)$ in $L_{\infty}(\Omega, A, P)$ is denoted similarly. Assume that for each $t \geqq 0,\left\|k_{0}(t, \tau ; \omega)\right\|$ and $\| k_{0}\left(t, \tau ; \omega\|\| \cdot\|x(\tau ; \omega)\|_{L_{2}(\Omega . A . P)}\right.$ are integrable with respect to $\tau \in R_{+}$for every $x(\tau ; \omega) \in C_{c}$.

Definition 2.1. We shall denote by $C_{g}$ the space of all continuous functions from $R_{+}$into $L_{2}(\Omega, A, P)$ such that, for $g(t)$ a positive, continuous function on $R_{+}$and some constant $Z>0$, we have

$$
\left\{\int_{\Omega}|x(t ; \omega)|^{2} d P(\omega)\right\}^{1 / 2} \leqq Z g(t), \quad t \geqq 0 .
$$

The norm in $C_{g}$ is defined by

$$
\|x(t ; \omega)\|_{C_{\vartheta}}=\sup _{t \in R_{+}}\left\{\frac{\|x(t ; \omega)\|_{L_{2}(\Omega . A . P)}}{g(t)}\right\} .
$$

Definition 2.2. Let $C$ denote the space of all continuous and bounded functions from $R_{+}$into $L_{2}(\Omega, A, P)$.

Definition 2.3. By $C_{c}=C_{c}\left(R_{+}, L_{2}(\Omega, A, P)\right)$ we mean the space of all continuous functions from $R_{+}$into $L_{2}(\Omega, A, P)$ with the topology of uniform convergence on every interval $[0, Q], Q>0$.

Let $B$ and $D$ be a pair of Banach spaces such that $B, D \subset C_{c}$, and let $T$ be a linear operator from $C_{c}$ into itself.

Definition 2.4. The pair of Banach spaces $(B, D)$ is said to be admissible with respect to $T: C_{c} \rightarrow C_{c}$ if and only if $T(B) \subset D$.

Definition 2.5. The Banach space $B$ is said to be stronger than $C_{c}$ if every convergent sequence in $B$ with respect to its norm also converges in $C_{c}$, but the converse is not true in general.

Let $H \subset C_{c}$ be the space of all functions such that the inner product in $L_{2}(\Omega, A, P)$ of an element $x(t ; \omega)$ with itself is integrable on $R_{+}$, i.e.

$$
\|x(t ; \omega)\|_{L_{2}(\Omega, A, P)}^{2}=\int_{\Omega}|x(t ; \omega)|^{2} d P(\omega), \quad t \in R_{+},
$$

and $y(t ; \omega) \in H$ if its inner product with itself and with any $x(t ; \omega)$ for which (2.1) is integrable on $R_{+}$is integrable with respect to $t$, and let $H_{1}$ and $H_{2}$ be Hilbert spaces contained in $H$ with norms defined by

$$
\|x(t ; \omega)\|_{H_{i}}=\left\{\int_{0}^{\infty}\|x(t ; \omega)\|_{L_{2}(\Omega . A . P)}^{2} d t\right\}^{1 / 2}, \quad i=1,2 .
$$


By a random solution of a stochastic integral equation such as equation (1.1) we shall mean a random function $x(t ; \omega)$ which satisfies the equation $P$-a.e. In order to prove the following results, we employ the well-known fixed point theorems of Krasnosel'skir., Banach, and Schauder [4].

3. Main results. We now present theorems concerning the existence and uniqueness of random solutions of equations (1.1) and (1.2) which are the main results of the paper. We first state two lemmas which will be needed in the proofs.

LEMMA 3.1 (Tsokos [9]). Let $T$ be a continuous linear operator from $C_{c}\left(R_{+}, L_{2}(\Omega, A, P)\right)$ into itself. If $B$ and $D$ are Banach spaces stronger than $C_{f}\left(R_{+}, L_{2}(\Omega, A, P)\right)$ and if the pair $(B, D)$ is admissible with respect to $T$, then $T$ is continuous from $B$ into $D$.

The following lemma is similar to a theorem appearing in Schmeidler [8] and Yosida [10].

LEMMA 3.2. The integral operator $W_{M}$ defined from the Hilbert space $H_{2}$ into the Hilbert space $H_{1}$ by

$$
\left(W_{M} x\right)(t ; \omega)=\int_{0}^{M} k_{0}(t, \tau ; \omega) x(\tau ; \omega) d \tau,
$$

for $t \in[0, M]$, where $M$ is a positive integer, is a bounded, completely continuous operator if the stochastic kernel $k_{0}(t, \tau ; \omega)$ is such that $\int_{0}^{\infty} \int_{0}^{M} \| k_{0}\left(t, \tau ; \omega \|\left.!\right|^{2} d \tau d t\right.$ exists and is finite.

The proof follows the technique of Schmeidler [8, p. 45].

We shall first consider the random integral equation of the Fredholm type of the form

$$
x(t ; \omega)=h(t ; \omega)+\int_{0}^{M} k_{0}(t, \tau ; \omega) e(\tau, x(\tau ; \omega)) d \tau,
$$

for $t \in[0, M]$, where $M$ is a positive integer.

THEOREM 3.1. Consider the random integral equation (3.2) under the following conditions:

(i) $H_{1}$ and $H_{2}$ are Hilbert spaces stronger than $C_{c}$ such that the pair $\left(H_{2}, H_{1}\right)$ is admissible with respect to the linear operator $W_{M}$ given by equation (3.1) where $t \in[0, M], M$ is a positive integer, $k_{0}(t, \tau ; \omega)$ behaves as above, and $\int_{0}^{\infty} \int_{0}^{\infty} \| k_{0}\left(t, \tau ; \omega \|^{2} d \tau d t\right.$ exists and is finite;

(ii) $x(t ; \omega) \rightarrow e(t, x(t ; \omega))$ is a continuous operator on the set

$$
S=\left\{x(t ; \omega): x(t ; \omega) \in H_{1},\|x(t ; \omega)\|_{H_{1}} \leqq \rho\right\},
$$

with values in $H_{2}$ such that $\|e(t, x(t ; \omega))\|_{H_{2}} \leqq \gamma$, where $\gamma>0$ and $\rho>0$ are constants;

(iii) $h(t ; \omega) \in H_{1}$. 
Then there exists at least one random solution of equation (3.2), provided that $\|h(t ; \omega)\|_{H_{1}}+K_{M} \gamma \leqq \rho$, where $K_{M I}$ is the norm of the operator $W_{M}$.

Proof. Clearly, $S$ is a closed, bounded, convex subset of $H_{1}$, and $H_{1}$ and $H_{2}$ are Banach spaces, since they are Hilbert spaces.

Define the operator $V_{M I}$ from $S$ into $H_{1}$ by

$$
\left(V_{\lambda I} x\right)(t ; \omega)=h(t ; \omega)+\int_{0}^{M T} k_{0}(t, \tau ; \omega) e(\tau, x(\tau ; \omega)) d \tau
$$

for $t \in[0, M]$. From condition (i) of the theorem and Lemma 3.2 the operator $W_{M}$ is a completely continuous operator from $H_{2}$ into $H_{1}$. The function $e(t, x(t ; \omega))$ is a continuous and bounded mapping from $H_{1}$ into $H_{2}$ by condition (ii). We may express the operator $V_{M}$ as the composite $W_{M}$ e plus $h$, and therefore, $V_{M}$ is a completely continuous operator from $S$ into $H_{1}$ (Krasnosel'skiĭ [4]).

We must show that $V_{M I}(S) \subset S$. Let $x(t ; \omega) \in S$. Taking the norm of equation (3.3), we obtain

$$
\begin{aligned}
\left\|\left(V_{M I} x\right)(t ; \omega)\right\|_{H_{1}} & =\left\|h(t ; \omega)+\int_{0}^{. M} k_{0}(t, \tau ; \omega) e(\tau, x(\tau ; \omega)) d \tau\right\|_{H_{1}} \\
& \leqq\|h(t ; \omega)\|_{H_{1}}+K_{M}\|e(t, x(t ; \omega))\|_{H_{2}} \\
& \leqq\|h(t ; \omega)\|_{H_{1}}+K_{\lambda \hat{\gamma}} \leqq \rho
\end{aligned}
$$

by condition (ii) and the last hypothesis of the theorem. Hence, $\left(V_{M} x\right)(t ; \omega) \in S$.

Therefore, by Schauder's fixed-point theorem, there exists at least one random element $x(t ; \omega) \in S$ satisfying

$$
\begin{aligned}
x(t ; \omega) & =\left(V_{M I} x\right)(t ; \omega) \\
& =h(t ; \omega)+\int_{0}^{. I} k_{0}(t, \tau ; \omega) e(\tau, x(\tau ; \omega)) d \tau
\end{aligned}
$$

for $t \in[0, M]$, completing the proof.

The sequence of integral operators $W_{M}$ converges to the operator $W$ from $H_{2}$ into $H_{1}$ defined by

$$
(W x)(t ; \omega)=\int_{0}^{\infty} k_{0}(t, \tau ; \omega) x(\tau ; \omega) d \tau, \quad t \in R_{+},
$$

as $M \rightarrow \infty$. Then $W$ is completely continuous under the same condition as in (i) of Theorem 3.1. Hence, we have the following theorem.

THEOREM 3.2. Suppose the random integral equation (1.1) satisfies the following conditions:

(i) $H_{1}$ and $H_{2}$ are Hilbert spaces stronger than $C_{c}$ such that the pair $\left(H_{2}, H_{1}\right)$ is admissible with respect to the linear operator $W$ given by 
equation (3.4), where $k_{0}(t, \tau ; \omega)$ behaves as described above and $\int_{0}^{\infty} \int_{0}^{\infty}\left\|k_{0}(t, \tau ; \omega)\right\|^{2} d \tau d t$ exists and is finite;

(ii) Same as condition (ii) of Theorem 3.1;

(iii) Same as condition (iii) of Theorem 3.1.

Then there exists at least one random solution of equation (1.1) in $S$, provided $\|h(t ; \omega)\|_{H_{1}}+K \gamma \leqq \rho$, where $K$ is the norm of $W$.

The proof is similar to that of Theorem 3.1.

The following theorem concerns the existence of a unique random solution of the random integral equation (1.2).

THEOREM 3.3. Consider the random integral equation (1.2) subject to the following conditions:

(i) $B$ and $D$ are Banach spaces stronger than $C_{c}$ and $(B, D)$ is admissible with respect to each of the operators

and

$$
(T x)(t ; \omega)=\int_{0}^{t} k(t, \tau ; \omega) x(\tau ; \omega) d \tau
$$

$$
(W x)(t ; \omega)=\int_{0}^{\infty} k_{0}(t, \tau ; \omega) x(\tau ; \omega) d \tau,
$$

for $t \in R_{+}$, where $k(t, \tau ; \omega)$ and $k_{0}(i, \tau ; \omega)$ behave as above;

(ii) $x(t ; \omega) \rightarrow f(t, x(t ; \omega))$ is an operator on

$$
S=\left\{x(t ; \omega): x(t ; \omega) \in D,\|x(t ; \omega)\|_{D} \leqq \rho\right\}
$$

with values in B satisfying

$$
\|f(t, x(t ; \omega))-f(t, y(t ; \omega))\|_{B} \leqq \lambda\|x(t ; \omega)-y(t ; \omega)\|_{D}
$$

for $x(t ; \omega), y(t ; \omega) \in S$ and $\lambda$ a constant;

(iii) $x(t ; \omega) \rightarrow e(t, x(t ; \omega))$ is an operator on $S$ with values in $B$ satisfying

$$
\|e(t, x(t ; \omega))-e(t, y(t ; \omega))\|_{B} \leqq \xi\|x(t ; \omega)-y(t ; \omega)\|_{D}
$$

for $x(t ; \omega), y(t ; \omega) \in S$ and $\xi$ a constant

(iv) $h(t ; \omega) \in D$.

Then there exists a unique random solution of equation (1.2), provided

$$
\begin{gathered}
\lambda K_{1}+\xi K_{2}<1, \\
\|h(t ; \omega)\|_{D}+K_{1}\|f(t, 0)\|_{B}+K_{2}\|e(t, 0)\|_{B} \leqq \rho\left(1-\lambda K_{1}-\xi K_{2}\right),
\end{gathered}
$$

where $K_{1}$ and $K_{2}$ are the norms of $T$ and $W$, respectively.

Proof. The operators $T$ and $W$ are continuous operators from $B$ into $D$ by the continuity and integrability assumptions on $k(t, \tau ; \omega)$ and 
$k_{0}(t, \tau ; \omega)$, condition (i), and Lemma 3.1. Therefore, $T$ and $W$ are bounded operators.

Define the operators $U$ and $V$ from $S$ into $D$ by

$$
\begin{aligned}
& (U x)(t ; \omega)=h(t ; \omega)+\int_{0}^{t} k(t, \tau ; \omega) f(\tau, x(\tau ; \omega)) d \tau, \\
& (V x)(t ; \omega)=\int_{0}^{\infty} k_{0}(t, \tau ; \omega) e(\tau, x(\tau ; \omega)) d \tau, \quad t \geqq 0 .
\end{aligned}
$$

We show that the conditions of Banach's fixed-point theorem hold. That is, $(U x)(t ; \omega)+(V x)(t ; \omega) \in S$ whenever $x(t ; \omega) \in S$, and $U+V$ is a contraction operator on $S$.

Let $x(t ; \omega), y(t ; \omega) \in S$. Then

$$
\begin{aligned}
\|(U x)(t ; \omega)+ & (V x)(t ; \omega) \|_{D} \\
& =\| h(t ; \omega)+\int_{0}^{t} k(t, \tau ; \omega) f(\tau, x(\tau ; \omega)) d \tau \\
& \quad+\int_{0}^{\infty} k_{0}(t, \tau ; \omega) e(\tau, x(\tau ; \omega)) d \tau \|_{D} \\
& \leqq\|h(t ; \omega)\|_{D}+K_{1}\|f(t, x(t ; \omega))\|_{B}+K_{2}\|e(t, x(t ; \omega))\|_{B} .
\end{aligned}
$$

But from condition (ii) $\|f(t, x(t ; \omega))\|_{B} \leqq \lambda\|x(t ; \omega)-0\|_{D}+\|f(t, 0)\|_{B}$, and from condition (iii) a similar inequality holds for $e(t, x(t ; \omega))$. Thus,

$$
\begin{aligned}
& \|(U x)(t ; \omega)+(V x)(t ; \omega)\|_{D} \\
& \leqq\|h(t ; \omega)\|_{D}+K_{1}\|f(t, 0)\|_{B}+\lambda K_{1} \rho+K_{2}\|e(t, 0)\|_{B}+\xi K_{2} \rho \\
& \leqq \rho\left(1-\lambda K_{1}-\xi K_{2}\right)+\lambda K_{1} \rho+\xi K_{2} \rho=\rho
\end{aligned}
$$

by the last hypothesis of the theorem. So $(U x)(t ; \omega)+(V x)(t ; \omega) \in S$.

Also,

$$
\begin{aligned}
\|(U x)(t ; \omega)+ & (V x)(t ; \omega)-(U y)(t ; \omega)-(V y)(t ; \omega) \|_{D} . \\
= & \| \int_{0}^{t} k(t, \tau ; \omega)[f(\tau, x(\tau ; \omega))-f(\tau, y(\tau ; \omega))] d \tau \\
& \quad \quad \quad+\int_{0}^{\infty} k_{0}(t, \tau ; \omega)[e(\tau, x(\tau ; \omega))-e(\tau, y(\tau ; \omega))] d \tau \|_{D} \\
\leqq & K_{1}\|f(t, x(t ; \omega))-f(t, y(t ; \omega))\|_{B} \\
& \quad+K_{2}\|e(t, x(t ; \omega))-e(t, y(t ; \omega))\|_{B} \\
\leqq & \left(\lambda K_{1}+\xi K_{2}\right)\|x(t ; \omega)-y(t ; \omega)\|_{D}
\end{aligned}
$$

by condition (ii). Since $\lambda K_{1}+\xi K_{2}<1$ by hypothesis, $U+V$ is a contraction mapping on $S$. 
Therefore, by Banach's fixed-point theorem there exists a unique element $x(t ; \omega) \in S$ satisfying

$$
x(t ; \omega)=(U x)(t ; \omega)+(V x)(t ; \omega),
$$

that is, a unique random solution of equation (1.2) exists, completing the proof.

For the case that $W$ is the null operator, we obtain the main theorem of Tsokos [9]. For the case that $T$ is the null operator, we obtain equation (1.1), and in this instance the above theorem gives conditions under which a unique random solution of equation (1.1) exists.

Several special cases of the above results may be obtained by taking as the spaces $B$ and $D$ the spaces $C_{g}$ or $C$.

THEOREM 3.4. Suppose the random integral equation (1.1) satisfies the following conditions:

(i) There exists a constant $Z>0$ and a positive continuous function $g(t)$ on $R_{+}$such that

$$
\int_{0}^{\infty}\left\|k_{0}(t, \tau ; \omega)\right\| g(\tau) d \tau \leqq Z, \quad t \in R_{+} ;
$$

(ii) $e(t, x)$ is continuous in $t \in R_{+}$uniformly in $x \in R$ such that $|e(t, 0)| \leqq$ $\gamma g(t)$ and

$$
|e(t, x)-e(t, y)| \leqq \xi g(t)|x-y|
$$

for $\|x\|_{C},\|y\|_{C} \leqq \rho$ and $\gamma>0$ and $\xi>0$ constants;

(iii) $h(t ; \omega) \in C$.

Then there exists a unique random solution, $x(t ; \omega) \in C$, such that $\|x(t ; \omega)\|_{C} \leqq \rho$, provided that $\|h(t ; \omega)\|_{C}, \xi$, and $\gamma$ are sufficiently small.

Proof. If we show that the pair $\left(C_{g}, C\right)$ is admissible with respect to the operator $W$ given by equation (3.4), then we are through, since the other conditions are equivalent to those of Theorem 3.3.

Let $x(t ; \omega) \in C_{g}$. Then

$$
\begin{aligned}
\|(W x)(t ; \omega)\|_{L_{2}(\Omega, A, P)} & \leqq \int_{0}^{\infty}\left\|k_{0}(t, \tau ; \omega) x(\tau ; \omega)\right\|_{L_{2}(\Omega, A, P)} d \tau \\
& \leqq \int_{0}^{\infty}\left\|k_{0}(t, \tau ; \omega)\right\| \cdot \| \frac{\|x(\tau ; \omega)\|_{L_{\Omega}(\Omega, A, P)}}{g(\tau)} g(\tau) d \tau \\
& \leqq \sup _{t \geq 0}\left\{\frac{\|x(t ; \omega)\|_{L_{2}(\Omega, A, P)}}{g(t)}\right\} \int_{0}^{\infty}\left\|k_{0}(t, \tau ; \omega)\right\| g(\tau) d \tau \\
& \leqq\|x(t ; \omega)\|_{C_{\theta}} Z,
\end{aligned}
$$


by condition (i) and the definition of the norm in the space $C_{g}$. Hence, $(W x)(t ; \omega)$ is in $C$ whenever $x(t ; \omega) \in C_{g}$, and the pair $\left(C_{g}, C\right)$ is admissible with respect to $W$, completing the proof.

We also obtain the following corollary.

COROLlaRY 3.1. Assume that the random integral equation (1.1) satisfies the following conditions:

(i) $\left\|k_{0}(t, \tau ; \omega)\right\| \leqq \Lambda, t, \tau \in R_{+}$, and $\int_{0}^{\infty} g(t) d t<\infty$, where $\Lambda>0$ is a constant;

(ii) Same as Theorem 3.4 (ii);

(iii) Same as Theorem 3.4 (iii).

Then there exists a unique random solution of (1.1) bounded on $R_{+}$, provided that $\|h(t ; \omega)\|_{C}, \xi$, and $\gamma$ are small enough.

The proof consists of showing that $\left(C_{g}, C\right)$ is admissible under (i) as in Theorem 3.4.

A comprehensive study of the stochastic integral equation (1.2) will appear in a later paper of the authors [6].

\section{BIBLIOGRAPHY}

1. M. W. Anderson, Stochastic integral equations, Ph.D. Dissertation, University of Tennessee, 1966.

2. C. Corduneanu, Problèmes globaux dans la théorie des équations intégrales de Volterra, Ann. Mat. Pura Appl. (4) 67 (1965), 349-363. MR 32 \#331.

3. - Some perturbation problems in the theory of integral equations, Math. Systems Theory 1 (1967), 143-155. MR 35 \#4773.

4. M. A. Krasnosel'skii, Topological methods in the theory of nonlinear integral equations, GITTL, Moscow, 1956; English transl., Macmillan, New York, 1964. MR 20 \#3464; MR 28 \#2414.

5. R. K. Miller, J. A. Nohel and J. S. W. Wong, A stability theorem for nonlinear mixed integral equations, J. Math. Anal. Appl. 25 (1969), 446-449. MR 38 \#2556.

6. W. J. Padgett and C. P. Tsokos, On a stochastic integral equation of the Fredholm type, Z. Wahrscheinlichkeitstheorie und Verw. Gebiete (to appear).

7. D. Petrovanu, Équations Hammerstein intégrales et discrètes, Ann. Mat. Pura Appl. (4) 74 (1966), 227-254. MR 35 \#687.

8. W. Schmeidler, Linear operations in Hilbert space, Academic Press, New York, 1965. MR 32 \#363.

9. C. P. Tsokos, On a stochastic integral equation of the Volterra type, Math. Systems Theory 3 (1969), 222-231. MR 40 \#949.

10. K. Yosida, Functional analysis, Die Grundlehren der math. Wissenschaften, Band 123, Academic Press, New York; Springer-Verlag, Berlin, 1965. MR 31 \#5054.

Department of Statistics, Virginia Polytechnic Institute and State UNIVERSITY, BLACKSBURG, VIRGINIA 24061

Current address (W. J. Padgett): Department of Mathematics, University of South Carolina, Columbia, South Carolina 29208 